\title{
Phenomenology of the gauge symmetry for right-handed fermions
}

\author{
Wei Chao $^{\mathrm{a}}$ \\ Center for Advanced Quantum Studies, Department of Physics, Beijing Normal University, Beijing 100875, China
}

Received: 26 July 2017 / Accepted: 13 January 2018 / Published online: 5 February 2018

(C) The Author(s) 2018. This article is an open access publication

\begin{abstract}
In this paper we investigate the phenomenology of the U(1) gauge symmetry for right-handed fermions, where three right-handed neutrinos are introduced for anomalies cancellations. Constraints on the new gauge boson $Z_{\mathbf{R}}$ from $Z-Z^{\prime}$ mixing as well as the upper bound of $Z^{\prime}$ production cross section in di-lepton channel at the LHC are presented. We further study the neutrino mass and the phenomenology of $Z_{\mathbf{R}}$-portal dark matter in this model. The lightest right-handed neutrino can be the cold dark matter candidate stabilized by a $Z_{2}$ flavor symmetry. Our study shows that active neutrino masses can be generated via the modified type-II seesaw mechanism; right-handed neutrino is available dark matter candidate for its mass being very heavy, or for its mass at near the resonant regime of the SM Higgs and(or) the new bosons; constraint from the dilepton search at the $L H C$ is stronger than that from the $Z-Z^{\prime}$ mixing only for $g_{\mathbf{R}}<0.121$, where $g_{\mathbf{R}}$ is the new gauge coupling.
\end{abstract}

\section{Introduction}

Local U(1) extensions of the standard model (SM) are wellmotivated new physics at the $\mathrm{TeV}$-scale. It can be constructed either from the bottom-up approach, such as the flavorful gauge symmetry $U(1)_{\mathbf{L}_{i}-\mathbf{L}_{j}}$ [1], or from the topdown approach, such as $U(1)_{\mathbf{N}}$ [2] which comes from the spontaneous breaking of the $E_{6}$ grand unification theory. Many models with new U(1) gauge symmetry have been proposed addressing various problems, such as $U(1)_{\mathbf{L}_{i}-\mathbf{L}_{j}}$ [1], $U(1)_{\mathbf{N}}[2], U(1)_{\mathbf{B}-\mathbf{L}}[3-5], U(1)_{\mathbf{B}}[6,7], U(1)_{\mathbf{L}}[6-8]$, $U(1)_{\mathbf{B}+\mathbf{L}}[9,10]$ generic $\mathrm{U}(1)[11,12]$ etc. For a review of various $\mathrm{U}(1)$ models and collider signatures of the $\mathrm{U}(1)$-related gauge boson, we refer the reader to Ref. [13] for detail. Of various local $\mathrm{U}(1)$ models, the $U(1)_{\mathbf{R}}$, a gauge symmetry for right-handed fermions, is attractive for following reasons:

\footnotetext{
a e-mail: chaowei@bnu.edu.cn
}

- its anomalies can be easily cancelled by introducing three right-handed Majorana neutrinos only,

- it may occur in left-right symmetric models [14] and in SO(10) models $[15,16]$,

- it may solve the vacuum stability problem [17] without appealing to for extra Higgs interactions,

but its phenomenology was not studied in detail in any reference.

In this paper phenomenology relevant to the $U(1)_{\mathbf{R}}$ is investigated. We first focus on constraint on the model from $Z-Z^{\prime}$ mixing since there is tree-level mixing between $Z$ and $Z^{\prime}$ in the local $U(1)_{\mathbf{R}}$ model. Mixing angles of the $Z_{\mathbf{R}}$ with $Z$ and $\gamma$ as well as the mass spectrum of gauge bosons are calculated. It shows that the $Z-Z^{\prime}$ mixing puts a lower bound on $v_{\Phi} / v$, which is the function of $g_{\mathbf{R}}$, where $v$ is the vacuum expectation value (VEV) of the SM Higgs, $v_{\Phi}$ is the VEV of the scalar singlet $\Phi$ that breaks the $U(1)_{\mathbf{R}}$ spontaneously, $g_{\mathbf{R}}$ is the gauge coupling of $U(1)_{\mathbf{R}}$, while the precisely measured $Z$ boson mass puts a stronger constraint on $v_{\Phi} / v: v_{\Phi} / v>73.32$.

Then we study how to naturally realize neutrino masses in the $U(1)_{\mathbf{R}}$ model. The discovery of the neutrino oscillations has confirmed that neutrinos are massive and lepton flavors are mixed, which provides the first evidence for new physics beyond the SM. Canonical seesaw mechanisms [18-30] provide a natural way in understanding the tiny but non-zero neutrino masses. In the $U(1)_{\mathbf{R}}$ model the mass matrix of righthanded Majorana neutrinos originates from their Yukawa couplings with $\Phi$, and their masses are thus at the TeV-scale, such that it predicts a tiny Yukawa coupling of right-handed neutrinos with left-handed lepton doublets if active neutrino masses are generated from the type-I seesaw mechanism. We show that neutrino masses can be generated from the modified type-II seesaw mechanism, where the scalar triplet carries no $U(1)_{\mathbf{R}}$ charge and its coupling with other scalars breaks the $\mathbf{B}-\mathbf{L}$ explicitly.

After that we focus on the phenomenology of $Z_{\mathbf{R}}$-portal dark matter. The fact that about $26.8 \%$ of the universe is 
made of dark matter with relic abundance $\Omega h^{2}=0.1189$, has been firmly established, while the nature of the dark matter is still unclear. Imposing the $Z_{2}$-symmetry on right-handed neutrinos only, the lightest right-handed neutrino $\mathbf{N}$ can be cold dark matter candidate. We study constraint on the model from the observed dark matter relic density, the exclusion limits of the spin-independent direct detection cross section mediated by $\Phi$ and $H$, as well as the spin-dependent direct detection cross section mediated by the $Z_{\mathbf{R}}$. It shows that right-handed neutrino dark matter is available only for its mass at near the resonance of SM Higgs, new scalar singlet and the $Z_{\mathbf{R}}$, or for its mass being very heavy.

Finally we investigate collider signatures of the $Z_{\mathbf{R}}$ at the LHC.Comparing its production cross section at the LHC the with upper limits given by the ATLAS, we get the lower limit on the $Z_{\mathbf{R}}$ mass, which is the function of $g_{\mathbf{R}}$. It shows that the constraint from the dilepton search at the LHC is stronger than that from the $Z-Z_{\mathbf{R}}$ mixing only for $g_{\mathbf{R}}<0.121$.

The paper is organized as follows: In Sect. 2 we introduce the model in detail. In Sect. 3 we study constraint on the model from $Z-Z^{\prime}$ mixing. Sections. 4 and 5 are focused on the neutrino mass and the dark matter phenomenology, respectively. We study the collider signature of $Z_{\mathbf{R}}$ in Sect. 6 . The last part is concluding remarks.

\section{The model}

We formulate our model in this section. Only right-handed fermions and the SM Higgs carry non-zero $U(1)_{\mathbf{R}}$ charge which we normalize to be multiples of " $\beta$ ". The SM provides the even number of fermion doublets required by the global $S U(2)$ anomaly [31]. The absence of axial-vector anomalies [32-34] and the gravitational-gauge anomaly [35-37] require that the SM should be extended with three righthanded neutrinos. We list in Table 1 the quantum number of various fields under the $U(1)_{\mathbf{R}}$. The anomaly cancellation conditions are listed in Table 2 . In the following studies, we set $\beta=1$ for simplicity.

The covariant derivative can be written as $D_{\mu}=\partial_{\mu}-$ $i g \tau^{a} A_{\mu}^{a}-i g^{\prime} Y B_{\mu}-i g_{\mathbf{R}} Y_{\mathbf{R}} B_{\mu}^{\prime}$, where $g_{\mathbf{R}}$ is the gauge coupling of $U(1)_{\mathbf{R}}$ and $Y_{\mathbf{R}}=0, \pm 1$. The scalar potential and the Yukawa interactions take the following form:

Table 1 Quantum numbers of various fields under the local $U(1)_{\mathbf{R}}, \ell_{L}$ and $Q_{L}$ are left-handed fermion doublets, $\mathbf{N}_{R}$ is right-handed neutrinos, $\Phi$ is the electroweak singlet scalar

\begin{tabular}{lllllllll}
\hline Fields & $\ell$ & $Q_{L}$ & $\mathbf{N}_{R}$ & $E_{R}$ & $U_{R}$ & $D_{R}$ & $H$ & $\Phi$ \\
\hline$U(1)_{\mathbf{R}}$ & 0 & 0 & $\beta$ & $-\beta$ & $\beta$ & $-\beta$ & $\beta$ & $-2 \beta$ \\
$Z_{2}$ & + & + & - & + & + & + & + & + \\
\hline
\end{tabular}

Table 2 The anomaly cancellation conditions of the $U(1)_{\mathbf{R}}$

\begin{tabular}{ll}
\hline Anomalies & Anomaly free conditions \\
\hline$S U(3)_{C}^{2} U(1)_{\mathbf{R}}$ & $-2(\beta)-2(-\beta)=0$ \\
$U(1)_{Y}^{2} U(1)_{\mathbf{R}}$ & $-\left[3\left(\frac{2}{3}\right)^{2} \beta+3\left(\frac{1}{3}\right)^{2}(-\beta)+(-1)^{2}(-\beta)\right]=0$ \\
$U(1)_{\mathbf{R}}^{2} U(1)_{Y}$ & $-\beta^{2}\left[3 \times \frac{2}{3}-3 \times \frac{1}{3}-1\right]=0$ \\
$U(1)_{\mathbf{R}}$ & $-[\beta+(-\beta)]-3[\beta+(-\beta)]=0$ \\
$U(1)_{\mathbf{R}}^{3}$ & $-\left[\beta^{3}+(-\beta)^{3}\right]-3\left[\beta^{3}+(-\beta)^{3}\right]=0$ \\
\hline
\end{tabular}

$$
\begin{aligned}
V= & -\mu^{2} H^{\dagger} H+\lambda\left(H^{\dagger} H\right)^{2}-\mu_{\Phi}^{2} \Phi^{\dagger} \Phi+\lambda_{1}\left(\Phi^{\dagger} \Phi\right)^{2} \\
& +\lambda_{2}\left(\Phi^{\dagger} \Phi\right)\left(H^{\dagger} H\right) \\
\mathcal{L}_{Y}= & -\overline{Q_{L}} Y_{U} \widetilde{H} U_{R}-\overline{Q_{L}} Y_{D} H D_{R} \\
& -\overline{\ell_{L}} Y_{E} H E_{R}-\overline{\mathbf{N}_{R}^{C}} Y_{\mathbf{N}} \Phi \mathbf{N}_{R}+\text { h.c. }
\end{aligned}
$$

where $H \equiv\left[G^{+},\left(h+i G_{h}^{0}+v\right) / \sqrt{2}\right]^{T}$ with $v$ the VEV of $H$ and $\Phi \equiv\left(\phi+i G_{S}^{0}+v_{\Phi}\right) / \sqrt{2}$ with $v_{\Phi}$ the VEV of $\Phi, Y_{U}, Y_{D}$ and $Y_{E}$ are $3 \times 3$ Yukawa matrices. The Yukawa interaction of right-handed neutrinos with left-handed lepton doublets is forbidden by the $Z_{2}$ discrete flavor symmetry and the lightest $\mathbf{N}_{R}$ is the cold dark matter candidate. Imposing the minimization conditions, one has

$$
\begin{aligned}
m_{h, \phi}^{2} & =\left(v^{2} \lambda+v_{\Phi}^{2} \lambda_{1}\right) \mp \sqrt{\left(v^{2} \lambda-v_{\Phi}^{2} \lambda_{1}\right)^{2}+v^{2} v_{\Phi}^{2} \lambda_{2}^{2}} \\
\alpha & =\frac{1}{2} \arctan \left[\frac{v v_{\Phi} \lambda_{2}}{v^{2} \lambda-v_{\Phi}^{2} \lambda_{1}}\right]
\end{aligned}
$$

where $\alpha$ is the mixing angle between CP even scalars. The physical parameters in the scalar sector are $m_{h}, m_{\phi}, \alpha, v$ and $v_{\Phi}$, while all other parameters can be reconstructed by them. The mixing angle $\alpha$ is constrained by the data from Higgs measurements at the LHC. Universal Higgs fits [38] to the data of ATLAS and CMS collaborations were performed in Refs. [9,10,39], and one has $\cos \alpha>0.865$ at the $95 \%$ confidence level. The constraint from electroweak precision observables is usually weaker than that from universal Higgs fit [40]. For the beta functions of $\lambda_{i}$ and $g_{\mathbf{R}}$ as well as their impacts on the vacuum stability of the SM Higgs, we refer the reader to Ref. [17] for detail, where the stability of the $\mathrm{SM}$ vacuum can be enhanced by the new gauge coupling.

\section{Vector boson Masses and Mixings}

Masses of gauge bosons come from the spontaneous breaking of the $S U(2)_{L} \times U(1)_{Y} \times U(1)_{\mathbf{R}}$ gauge symmetry. Since the SM Higgs carries non-zero $U(1)_{\mathbf{R}}$ charge, there is mixing between $Z$ and $Z_{\mathbf{R}}$ at the tree-level. ${ }^{1}$ The mass matrix of

\footnotetext{
$\overline{1}$ There might be kinematic mixing term $\varepsilon B^{\mu v} B_{\mu \nu}^{\prime}$ in the Lagrangian, which may exist in any $\mathrm{U}(1)$ extension of the SM and may lead to the
} 

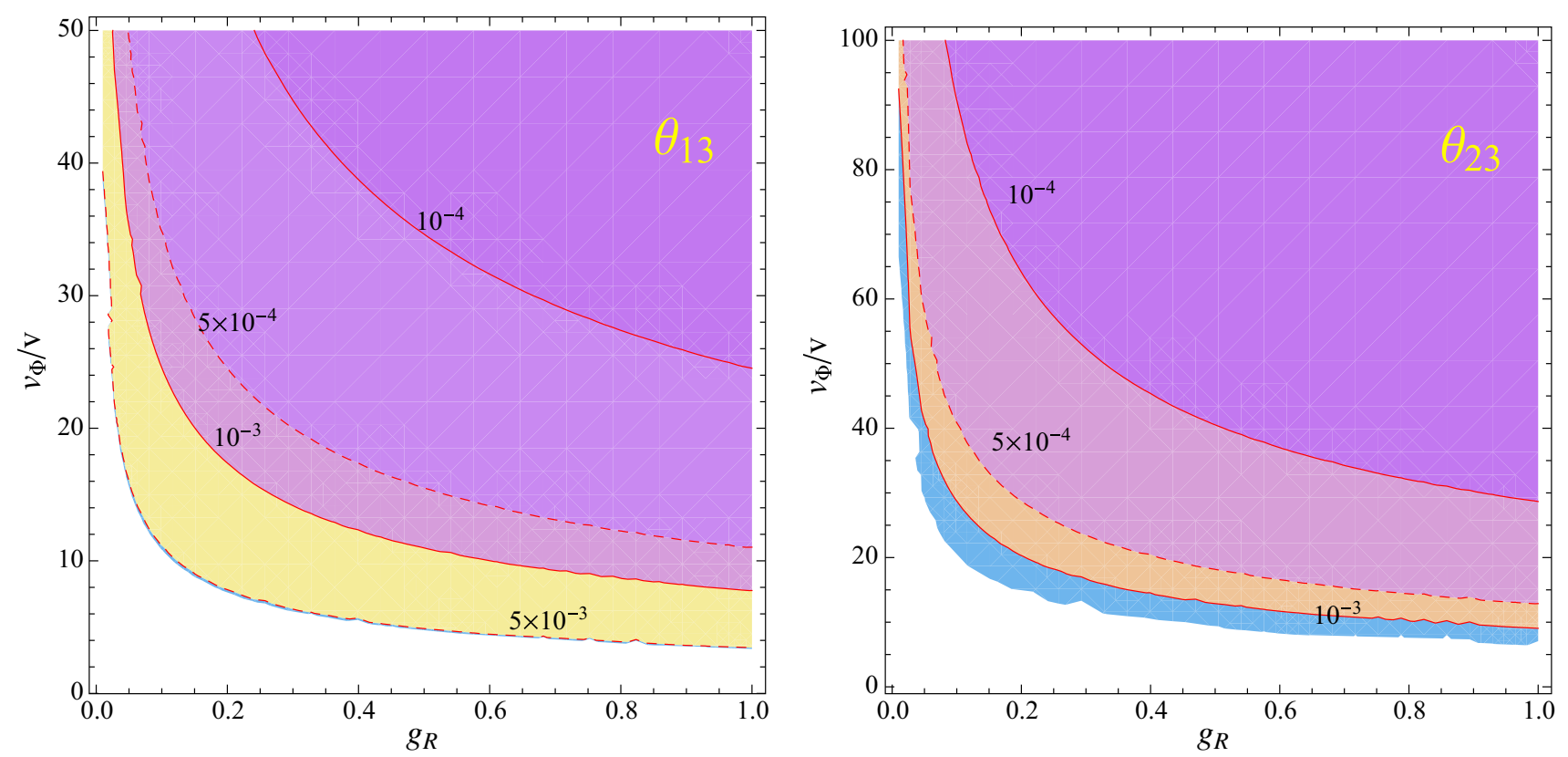

Fig. 1 Contours of $\theta_{13}$ (left-panel) and $\theta_{23}$ (right-panel) in the $g_{\mathbf{R}}-v_{\Phi} / v$ plane

neutral vector bosons in the basis $\left(B_{\mu}, W_{\mu}^{3}, B_{\mu}^{\prime}\right)$ is given by:

$M_{V}^{2}=\frac{v^{2}}{4}\left(\begin{array}{lll}g^{\prime 2} & -g^{\prime} g & 2 g^{\prime} g_{\mathbf{R}} \\ -g^{\prime} g & g^{2} & -2 g g_{\mathbf{R}} \\ 2 g^{\prime} g_{\mathbf{R}} & -2 g g_{\mathbf{R}} & 4 g_{\mathbf{R}}^{2}(1+\delta)\end{array}\right)$

where $g, g^{\prime}$ are gauge couplings of $S U(2)_{L}$ and $U(1)_{Y}$ respectively; $\delta=4 v_{\Phi}^{2} / v^{2}$. In the limit $g_{\mathbf{R}} \rightarrow 0$, one gets the mass matrix of the SM gauge bosons. The mass matrix in Eq. (5) can be diagonalized by the $3 \times 3$ unitary transformation, $\mathcal{U}^{\dagger} M_{V}^{2} \mathcal{U}^{*}=\operatorname{diag}\left\{0, M_{Z}^{2}, M_{Z_{\mathbf{R}}}^{2}\right\}$, where $\mathcal{U}$ can be written in terms of the standard parameterization:

$\mathcal{U}=\left(\begin{array}{lll}c_{12} c_{13} & s_{12} c_{13} & c_{13} \\ -c_{23} s_{12}-c_{12} s_{13} s_{23} & c_{12} c_{23}-s_{12} s_{13} s_{23} & c_{13} s_{23} \\ -c_{12} c_{23} s_{13}+s_{12} s_{23} & -c_{23} s_{12} s_{13}-c_{12} s_{23} & c_{13} c_{23}\end{array}\right)$

with $c_{i j} \equiv \cos \theta_{i j}$ and $s_{i j} \equiv \sin \theta_{i j}$. Mass eigenvalues of $Z$ and $Z_{\mathbf{R}}$ are

$$
\begin{aligned}
& M_{Z_{\mathbf{R}}(Z)}^{2}=\frac{v^{2}}{8}\left\{g^{2}+g^{\prime 2}+4 g_{\mathbf{R}}^{2}(1+\delta)\right. \\
& \left. \pm \sqrt{-16 \delta\left(g^{2}+g^{\prime 2}\right) g_{\mathbf{R}}^{2}+\left[g^{2}+g^{\prime 2}+4 g_{\mathbf{R}}^{2}(1+\delta)\right]^{2}}\right\}
\end{aligned}
$$

Footnote 1 continued

mixing between neutral gauge bosons. Here we assume $\varepsilon \rightarrow 0$, because the tree-level mixing is the key feature that discriminate our model from other U(1)s. Furthermore, $Z-Z^{\prime}$ mixing may arise at loop-level, which is subdominant and is thus neglected. For expressions of loop induced $Z-Z^{\prime}$ mixing, we refer the reader to the appendix of our earlier paper Ref. [52] for detail.
For the case $4 g_{\mathbf{R}}^{2}(1+\delta) \gg g^{2}+g^{\prime 2}$, which corresponds to the decoupled limit, it has $M_{Z^{\prime}}^{2} \approx v^{2}(1+\delta) g_{\mathbf{R}}^{2}$ and $M_{Z}^{2} \approx$ $\left(g^{2}+g^{\prime 2}\right) v^{2} / 4$. Mixing angles are actually the function of $g_{\mathbf{R}}$ and $\delta$ :

$$
\begin{aligned}
\tan \theta_{23} & =-\frac{g}{2 g_{\mathbf{R}}}\left(1-\frac{g_{\mathbf{R}}^{2} v^{2} \delta}{M_{Z_{\mathbf{R}}}^{2}}\right) \\
\sin \theta_{13} & =\frac{g^{\prime}\left(M_{Z_{\mathbf{R}}}^{2}-g_{\mathbf{R}}^{2} v^{2} \delta\right)}{\left[\left(g^{2}+g^{\prime 2}\right)\left(M_{Z_{\mathbf{R}}}^{2}-g_{\mathbf{R}}^{2} v^{2} \delta\right)^{2}+4 g_{\mathbf{R}}^{2} M_{Z_{\mathbf{R}}}^{4}\right]^{1 / 2}} \\
\tan \theta_{12} & =\frac{g^{\prime}\left(M_{Z}^{2}-g_{\mathbf{R}}^{2} v^{2} \delta\right) \sqrt{g^{2}+g^{\prime 2}}}{g\left[\left(g^{2}+g^{\prime 2}\right)\left(M_{Z}^{2}-g_{\mathbf{R}}^{2} v^{2} \delta\right)^{2}+4 g_{\mathbf{R}}^{2} M_{Z}^{4}\right]^{1 / 2}}
\end{aligned}
$$

where $\theta_{12}$ corresponds to the conventional weak mixing angle, $\theta_{13}$ and $\theta_{23}$ are mixing angles of $\gamma-Z_{\mathbf{R}}$ and $Z-Z_{\mathbf{R}}$ respectively.

Due to the $Z-Z_{\mathbf{R}}$ mixing, $Z_{\mathbf{R}}$ may decay into charged gauge boson pairs $W^{-} W^{+}$, which can be used to place constraint on the $Z-Z_{\mathbf{R}}$ mixing using diboson production at the LHC. It shows that the $Z-Z_{\mathbf{R}}$ mixing should be less than $0.7 \sim 2 \times 10^{-3}$ [41] derived from the data recoded by ATLAS and CMS collaborations at $\sqrt{s}=13 \mathrm{TeV}$ with integrated luminosities of $13.2 \mathrm{fb}^{-1}$ and $35.9 \mathrm{fb}^{-1}$, respectively. In Fig. 1, we show contours of the $\theta_{13}$ (leftpanel) and $\theta_{23}$ (right-panel) in the $g_{\mathbf{R}}-v_{\Phi} / v$ plane. We take $\alpha\left(M_{Z}\right)^{-1}=127.918, \sin ^{2} \theta_{W}\left(M_{Z}\right)=0.23122$ and $M_{Z}=91.1876 \mathrm{GeV}$ [42], which are used to get values of $g$ and $g^{\prime}$ respectively. One can see from the right-panel of Fig. 1 that, the scale of the $U(1)_{\mathbf{R}}$ breaking should be at least 


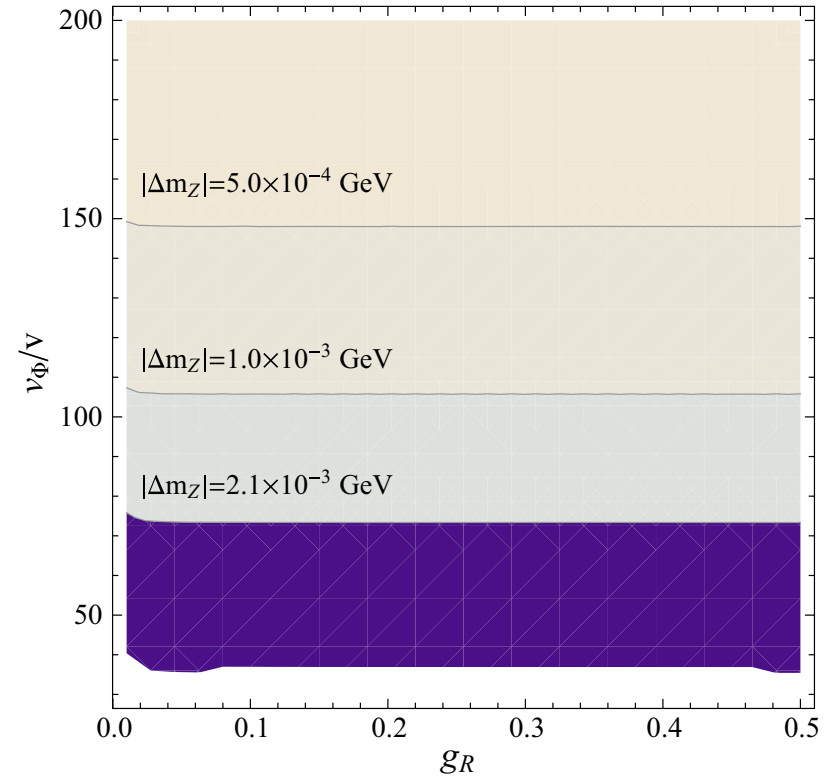

Fig. 2 Contours of $\Delta M_{Z}$ in the $g_{\mathbf{R}}-v_{\Phi} / v$ plane

one order higher than the electroweak scale. According to Eq. (7), the Z-boson mass is slightly changed in the $U(1)_{\mathbf{R}}$ model. There is thus constraint from the precision measurement of the $Z$ boson mass. We show in the Fig. 2 contours of $\Delta M_{Z}$, namely $M_{Z}-M_{Z}^{\text {observed }}$, in the $g_{\mathbf{R}}-v_{\Phi} / v$ plane. It shows that $\Delta M_{Z}$ is insensitive to the $g_{\mathbf{R}}$ when it is larger than 0.04. Using the ambiguity of the Z-boson mass given by the PDG [42], which is $\Delta M_{Z}<0.0021 \mathrm{GeV}$, one has $v_{\Phi} / v>73.32\left(v_{\Phi}>18 \mathrm{TeV}\right)$.

Before proceeding, we discuss how to distinguish the $U(1)_{\mathbf{R}}$ from the $U(1)_{\mathbf{B}-\mathbf{L}}$, which share many similarities. From previous study, it is clear that precision measurement of the $Z$ boson mass at the CEPC and (or) ILC can test the $U(1)_{\mathbf{R}}$, so here we mainly discuss how to distinguish them in colliders. First, $Z_{\mathbf{R}}$ couples to the SM Higgs, while there is no such interaction for the $Z^{\prime}$ of $U(1)_{\mathbf{B}-\mathbf{L}}$. The new interaction takes the form: $1 / 2 g_{R}^{2} Z_{\mathbf{R} \mu} Z_{\mathbf{R}}^{\mu}\left(h^{2}+2 h v\right)$, where the effect of $Z-Z_{\mathbf{R}}$ mixing is neglected. As a result, one can discriminate $U(1)_{\mathbf{R}}$ from $U(1)_{\mathbf{B}-\mathbf{L}}$ via the process $\bar{q} q \rightarrow Z_{\mathbf{R}_{-}}+h$, with $Z_{\mathbf{R}}$ decaying into di-lepton and $h$ decaying into $b \bar{b}$, in future $100 \mathrm{TeV}$ proton-proton collider. Second, $Z_{\mathbf{R}}$ only couple to right-handed fermions, while $Z^{\prime}$ of $U(1)_{\mathbf{B}-\mathbf{L}}$ couples to both left-handed and right-handed fermions. One can write down partially-polarized differential cross section for the process $e^{-} e^{+} \rightarrow Z_{\mathbf{R}}\left(Z^{\prime}\right) \rightarrow f \bar{f}$ at the CEPC and (or) ILC, with concrete helicity of initial electron(positron) and summed over the helicity of final states. It can then be applied to study the sensitivity to new gauge bosons through the measurement of a forward-backward asymmetry defined in Ref. [66] at lepton colliders. A detailed analysis will be out of the reach of this paper.

\section{Neutrino masses}

In this section we investigate how to generate Majorana masses of active neutrinos in the $U(1)_{\mathbf{R}}$ model. The solar, atmosphere, accelerator and reactor neutrino oscillation experiments have firmed that neutrinos are massive and lepton flavors are mixed. In our model right-handed neutrinos do not couple to left-handed lepton doublets, so that the conventional type-I seesaw mechanism does not work. We study the possibility of generating active neutrino masses via the type-II seesaw mechanism. Interactions relevant to the scalar triplet $\Delta$ with $Y=2$ can be written as

$$
\begin{aligned}
-\mathcal{L}_{\Delta}= & M_{\Delta}^{2} \Delta^{\dagger} \Delta+\left(\tilde{\lambda} H^{T} i \sigma_{2} \Delta H \Phi+\text { h.c. }\right) \\
& +\left(\overline{\ell_{L}} Y_{\Delta} \Delta \ell_{L}+\text { h.c. }\right)+\cdots
\end{aligned}
$$

where dots represent interactions of $\Delta$ that are irrelevant to the neutrino mass generation, $Y_{\Delta}$ is the symmetric Yukawa coupling matrix. Full expression of triplet interactions can be found in Ref. [43]. After the spontaneous breaking of the $S U(2)_{L} \times U(1)_{Y} \times U(1)_{\mathbf{R}}$ symmetry, the active neutrino masses can be written as

$$
M_{v}=Y_{\Delta} v_{\Delta} \approx Y_{\Delta} \tilde{\lambda} \frac{v^{2} v_{\Phi}}{M_{\Delta}^{2}}
$$

where $v_{\Delta}$ is the VEV of $\Delta$. The smallness of active neutrino masses is own to the tiny $v_{\Delta}$, which is suppressed by the $M_{\Delta}^{2}$. Notice that interactions in the first bracket of Eq. (11) breaks the lepton number $\mathbf{L}$ explicitly and the global $U(1)_{\mathbf{L}}$ can be recovered in the limit $\tilde{\lambda} \rightarrow 0$. According to the naturalness criterion [44], $\tilde{\lambda}$ should be naturally small. In this case $\Delta$ can be at the TeV scale and we refer the reader to Refs. [45-47] for signatures of $\Delta$ at the LHC.

\section{Dark matter}

About $26.8 \%$ of our universe is made of dark matter, whose relic abundance is given as $\Omega_{\mathrm{DM}} h^{2}=0.1198 \pm 0.0015$ [48]. Weakly Interacting Massive Particle (WIMP) [49] is a promising dark matter candidate, since the observed relic density can be naturally derived for a WIMP mass around the electroweak scale. In the $U(1)_{\mathbf{R}}$ model, the lightest righthanded Majorana neutrino can be cold dark matter candidate, stabilized by the $Z_{2}$ symmetry. ${ }^{2}$ We evaluate the relic abundance of the dark matter and study its implications in dark matter direct detections in this section. The dark matter $\mathbf{N}$ mainly couple to the $Z_{\mathbf{R}}, \hat{s}$ and $\hat{h}$, with Lagrangian:

$-\mathcal{L} \sim \frac{1}{2} \overline{\mathbf{N}} i g_{\mathbf{R}} \gamma_{\mu} \gamma_{5} Z_{\mathbf{R}}^{\mu} \mathbf{N}+\frac{1}{2} \frac{m_{\mathbf{N}}}{v_{\Phi}} \overline{\mathbf{N}}\left(c_{\alpha} \hat{s}-s_{\alpha} \hat{h}\right) \mathbf{N}$

\footnotetext{
${ }^{2}$ For the vector-boson portal neutrino dark matter, we refer the reader to Refs. [50-54] for detail.
} 
where $\mathbf{N}=N_{R}^{C}+N_{R}, m_{\mathbf{N}}$ is the mass eigenvalue of $\mathbf{N}, \hat{h}$ and $\hat{s}$ are the mass eigenstates of $h$ and $s$ respectively. The interaction of $\mathbf{N}$ with $Z$ can be neglected due to the tiny mixing angle $\theta_{23}{ }^{3}$

The evolution of the dark matter density $n$ is governed by the thermal average of reduced annihilation cross sections $\langle\sigma v\rangle$, which can be approximated with the non-relativistic expansion: $\langle\sigma v\rangle=a+b\left\langle v^{2}\right\rangle$. Contributions of various channels can be written as

$$
\begin{aligned}
& \langle\sigma v\rangle_{s_{a} s_{b}}=\frac{1}{1+\delta} \frac{\lambda^{1 / 2}\left(4, \zeta_{a}, \zeta_{b}\right)}{1024 \pi v_{\Phi}^{2} m_{\mathbf{N}}^{2}}\left|\frac{c_{\alpha} C_{s a b}}{4-\zeta_{s}}-\frac{s_{\alpha} C_{h a b}}{4-\zeta_{h}}\right|^{2}\left\langle v^{2}\right\rangle \\
& \langle\sigma v\rangle_{Z Z}=\frac{s_{2 \alpha}^{2} m_{Z}^{4}}{512 \pi m_{\mathbf{N}}^{2} v^{2} v_{\Phi}^{2}} \sqrt{1-\zeta_{Z}}\left(3-\frac{4}{\zeta_{Z}}+\frac{4}{\zeta_{Z}^{2}}\right) \\
& \left|\frac{1}{4-\zeta_{s}}-\frac{1}{4-\zeta_{h}}\right|^{2}\left\langle v^{2}\right\rangle, \\
& \langle\sigma v\rangle_{W W}=\frac{s_{2 \alpha}^{2} m_{W}^{4}}{256 \pi m_{\mathbf{N}}^{2} v^{2} v_{\Phi}^{2}} \sqrt{1-\zeta_{W}}\left(3-\frac{4}{\zeta_{W}}+\frac{4}{\zeta_{W}^{2}}\right) \\
& \left\langle\frac{1}{4-\zeta_{s}}-\left.\frac{1}{4-\zeta_{h}}\right|^{2}\left\langle v^{2}\right\rangle\right. \\
& \quad+\frac{n_{C}^{f} g_{\mathbf{R}}^{4}}{192 \pi m_{\mathbf{N}}^{2}} \sqrt{1-\zeta_{f}}\left(\zeta_{f}+2\right)\left|\frac{1}{4-\zeta_{Z_{\mathbf{R}}}}\right|^{2}\left\langle v^{2}\right\rangle+\frac{n_{C}^{f} g_{\mathbf{R}}^{4} \varrho_{Z_{\mathbf{R}}}^{f} \sqrt{1-\zeta_{f}}}{32 \pi m_{Z_{\mathbf{R}}}^{2}} \\
& \quad+\frac{23 \zeta_{f}^{2}-192 \varrho_{Z_{\mathbf{R}}}^{f} \zeta_{Z_{\mathbf{R}}}^{-1}+8\left(30 \varrho_{Z_{\mathbf{R}}}^{f 2}+12 \varrho_{Z_{\mathbf{R}}}^{f}+1\right)-4 \zeta_{f}\left(30 \varrho_{Z_{\mathbf{R}}}^{f}+7\right)}{768 \pi m_{\mathbf{N}}^{2} \sqrt{1-\zeta_{f}}} \\
& \quad \times \frac{n_{C}^{f} g_{\mathbf{R}}^{4}\left\langle v^{2}\right\rangle}{\left|4-\zeta_{Z_{\mathbf{R}}}\right|^{2}} \\
& \langle\sigma v\rangle_{Z^{\prime} s}=\frac{c_{\alpha}^{2} g_{\mathbf{R}^{2}}^{2} m_{Z^{\prime}}^{4}, \lambda^{3 / 2}\left(4, \zeta_{V}, \zeta_{s}\right)}{1024 \pi v_{\Phi}^{2} m_{\mathbf{N}}^{4} \zeta_{Z_{\mathbf{R}}}^{3}}+\mathcal{O}\left(\left\langle v^{2}\right\rangle\right) \\
& \hline n_{f}
\end{aligned}
$$

where $\lambda(x, y, z) \equiv x^{2}+y^{2}+z^{2}-2 x y-2 x z-2 y z$, $\zeta_{X}=m_{X}^{2} / m_{\mathbf{N}}^{2}, \varrho_{Z_{\mathbf{R}}}^{f}=m_{f}^{2} / m_{Z_{\mathbf{R}}}^{2} ; \delta_{a b}=1$ (for $a=b$ ) and 0 (for $a \neq b$ ); $C_{s_{i} s_{j} s_{k}}$ are trilinear couplings; $s_{2 \alpha}=\sin 2 \alpha$, $c_{\alpha}=\cos \alpha, n_{C}^{f}$ is the color factor of $f$.

The final relic density can be given by [55]

$\Omega_{\mathrm{DM}} h^{2}=\frac{1.07 \times 10^{9}}{M_{p l}} \frac{x_{F}}{\sqrt{g_{\star}}} \frac{1}{a+3 b / x_{F}}$

\footnotetext{
3 As was discussed in Sect. 3 the mixing between $Z_{\mathbf{R}}$ and the SM $Z$ bosons is constrained to be less than $\mathcal{O}(1) \times 10^{-3}$. As a result the process mediated by the $Z$-boson can be safely neglected in calculating dark matter relic density and constraints of direct detection, because its effects are at least two orders smaller than those of $Z_{\mathbf{R}}$ for its mass at the $\mathrm{TeV}$ scale.
}

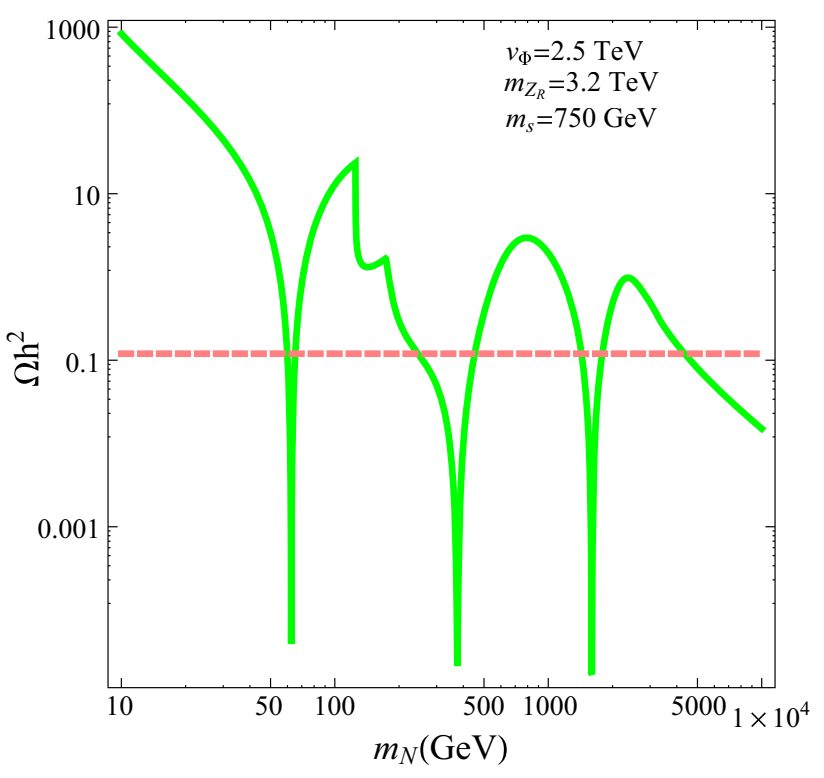

Fig. 3 The relic density of the dark matter as the function of the dark matter mass $m_{\mathbf{N}}$, by setting $c_{\alpha}=0.9, m_{s}=750 \mathrm{GeV}, v_{\Phi}=2.5 \mathrm{TeV}$ and $m_{Z_{\mathbf{R}}}=3.2 \mathrm{TeV}$. The horizontal line is the observed dark matter relic density. This plot is produced from the analytical results given in Eqs. (14)-(19)

where $M_{p l}=1.22 \times 10^{19} \mathrm{GeV}$ being the Planck mass, $x_{F}=m_{\mathbf{N}} / T_{F}$ with $T_{F}$ the freeze-out temperature, $g_{\star}$ is the effective degree of freedom at the freeze-out temperature. As an illustration, we show in Fig. 3 the dark matter relic density as the function of the dark matter mass $m_{\mathbf{N}}$ by setting $c_{\alpha}=0.9, m_{s}=750 \mathrm{GeV}, v_{\Phi}=2.5 \mathrm{TeV}$ and $m_{Z_{\mathbf{R}}}=3.2 \mathrm{TeV}$. The value of $g_{\mathbf{R}}$ is determined by the following formula $g_{\mathbf{R}} \approx M_{Z_{\mathbf{R}}} / \sqrt{v^{2}+4 v_{\mathbf{R}}^{2}}$. The horizontal line is the experimental value of the relic density. One can conclude from the plot that $\mathbf{N}$ is qualified dark matter when its mass lies near the resonance of $\hat{h}, \hat{s}$ or $Z_{\mathbf{R}}$ for low mass $\mathbf{N}$. For high dark matter mass regime, the relic density turns to be under-abundant since new annihilation channel is kinematically allowed. Thus heavy $\mathbf{N}$ is also good dark matter candidate.

The spin-independent $\mathbf{N}$-nucleus scattering cross section is

$\sigma_{\mathrm{SI}}=\frac{\mu^{2} s_{2 \alpha}^{2} m_{N}^{2}}{4 \pi v^{2} v_{\Phi}^{2}}\left(\frac{1}{m_{h}^{2}}-\frac{1}{m_{s}^{2}}\right)^{2}\left[Z f_{p}+(A-Z) f_{n}\right]^{2}$

where $\mu$ is the reduced mass of $\mathbf{N}$-nucleus system, $f_{p, n}=$ $m_{p, n}\left(2 / 9+7 / 9 \sum_{q=u, d, s} f_{T_{q}}^{p, n}\right)$ with $f_{T_{u}}^{p}=0.020 \pm 0.004$, $f_{T_{d}}^{p}=0.026 \pm 0.005, f_{T_{u}}^{n}=0.014 \pm 0.003, f_{T_{d}}^{n}=$ $0.036 \pm 0.008$ and $f_{T_{s}}^{p, n}=0.118 \pm 0.062$ [56]. We show in the left panel of Fig. 4 the rescaled spin-independent cross section, namely $\sigma_{\mathrm{SI}} \times \Omega_{\mathrm{DM}} h^{2} / 0.1189$, as the function of $m_{\mathbf{N}}$ by using the same inputs of the Fig. 3. We only focus on the 

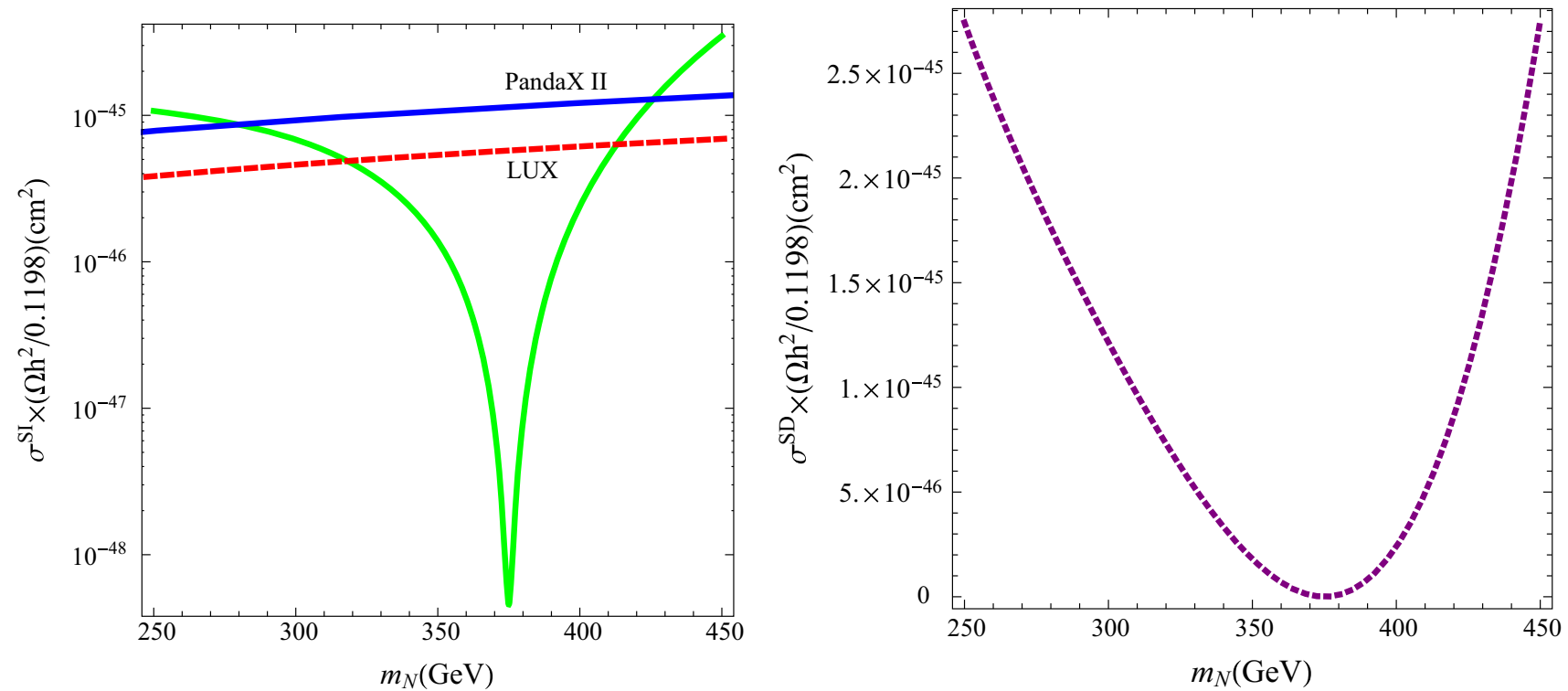

Fig. 4 The rescaled spin-independent cross section (left-panel) and spin-dependent cross section as the function of the dark matter mass, other inputs are the same as these in the Fig. 3. These plots originate from analytical results given in Eqs. (20) and (21)

resonant regime of $\hat{s}$ in this plot since there is redundancy relic density in other mass region and the resonant regime of $Z_{\mathbf{R}}(\hat{h})$ predicts a too heavy (light) dark matter. The reddashed and blue-solid lines are the exclusion limits of LUX 2016 [57] and PandaX II [58] respectively. The available parameter space is shrunk compared with the relic density allowed region.

Since $\mathbf{N}$ couples to $Z_{\mathbf{R}}$, there is spin-dependent cross section of $\mathbf{N}$ with nucleus which takes the following form

$\sigma^{\mathrm{SD}}=\frac{g_{\mathbf{R}}^{4} \mu^{2}}{\pi M_{Z_{\mathbf{R}}}^{4}}\left(\sum_{q=u, d, s} \lambda_{q}\right)^{2} J_{N}\left(J_{N}+1\right)$,

where $\lambda_{q}$ reduces to $\Delta^{p, n}$ for scattering off free proton or neutron with $\Delta_{u}^{p}=0.78 \pm 0.02, \Delta_{d}^{p}=-0.48 \pm 0.02, \Delta_{s}^{p}=$ $-0.15 \pm 0.02, \Delta_{u}^{n}=-0.48 \pm 0.02, \Delta_{d}^{n}=-0.78 \pm 0.02$, $\Delta_{s}^{n}=-0.15 \pm 0.02[56,59,60], J_{N}$ is the angular momentum of the nucleus and it equals to $1 / 2$ for free nucleons. We show in the right-panel of the Fig. 4 the rescaled spindependent cross section of $\mathbf{N}$ scattering off free neutron as the function of $m_{\mathbf{N}}$. The smallest excluded WIMP-neutron cross section is $\sigma_{n}^{\mathrm{SD}}=4.3 \times 10^{-41}$ at $m_{\mathrm{DM}}=45 \mathrm{GeV}$ from the PandaX-II [61]. One can conclude from the plot that the spin-dependent cross section at the resonant regime of $\hat{s}$ is much smaller than the current exclusion limit.

\section{Collider signatures}

In this section we investigate the signature of $Z_{\mathbf{R}}$ at the LHC. $Z_{\mathbf{R}}$ can be produced at the LHC via the Drell-Yan process, while the dilepton channel provides a significant signature.

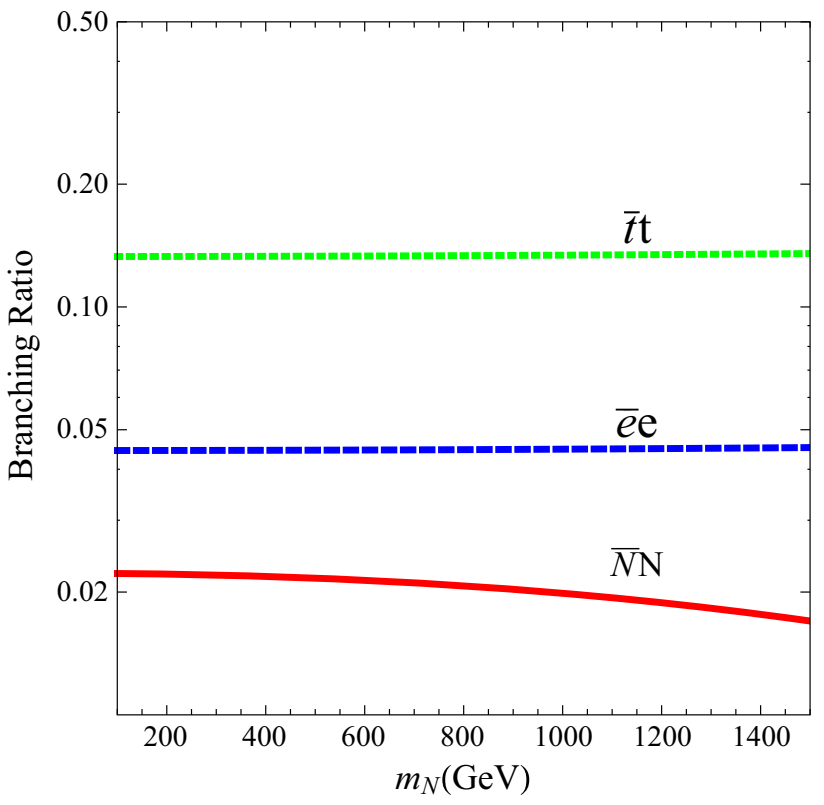

Fig. 5 Branching ratios of $Z_{\mathbf{R}}$ decaying into various final states

We first study the branching ratio of $Z_{\mathbf{R}}$. The decay rate of $Z_{\mathbf{R}}$ into fermion pairs can be written as

$\Gamma\left(\mathbf{Z}_{\mathbf{R}} \rightarrow \bar{f} f\right)=\frac{1}{1+\delta_{f}} \frac{n_{f}^{C} g_{\mathbf{R}}^{2} m_{\mathbf{Z}_{\mathbf{R}}}}{24 \pi}\left(1-\frac{m_{f}^{2}}{m_{\mathbf{Z}_{\mathbf{R}}}^{2}}\right)^{3 / 2}$,

where $\delta_{f}=1(0)$ if $f$ is (not) identical particle. $Z_{\mathbf{R}}$ can also decay into diboson pair $\left(W^{+} W^{-}\right)$due to its mixing with $Z$, which is suppressed by $\theta_{23}^{2}$ and will be neglected in the following analysis. We show in the Fig. 5 the branching ratio 


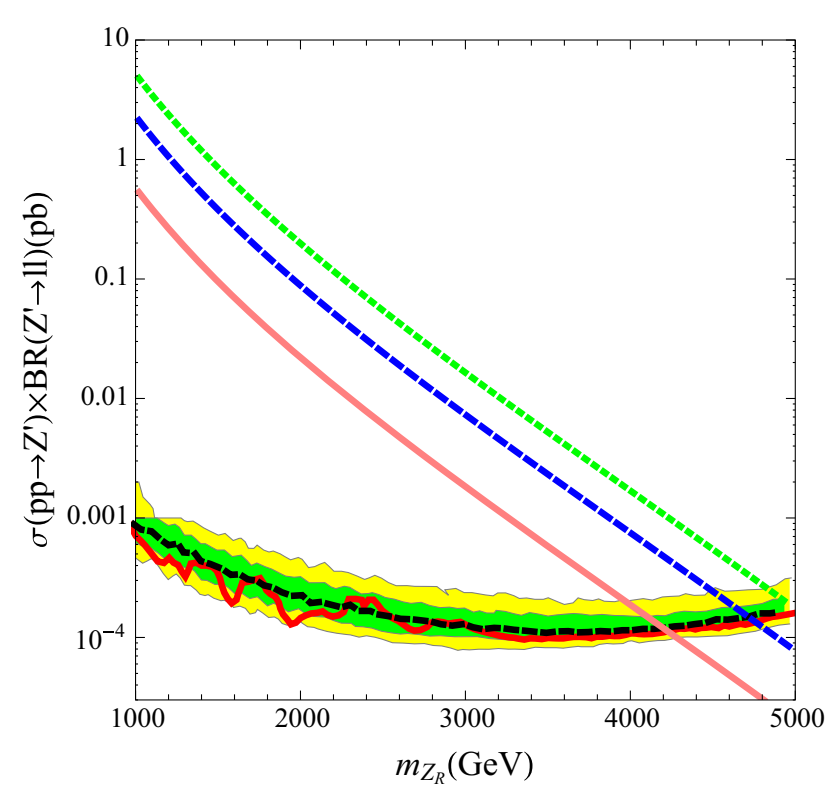

Fig. 6 Production cross section of process $\left(p p \rightarrow Z_{\mathbf{R}} \rightarrow \ell^{+} \ell^{-}\right)$as the function of $M_{Z_{\mathrm{R}}}$, the solid, dashed and dotted lines correspond to $g_{\mathbf{R}}=0.1,0.2$ and 0.3 respectively

of $Z_{\mathbf{R}}$ decaying into various final states as the function of $m_{\mathbf{N}}$ by setting $m_{Z_{\mathbf{R}}}=3.6 \mathrm{TeV}$, where the solid, dashed and dotted lines correspond to the decay channel of $\overline{\mathbf{N}} \mathbf{N}, \bar{e} e$ and $\bar{t} t$ respectively. It shows that the branching ratio of dilepton with discrete flavor is about $4.5 \%$.

The cross section of the process $p p \rightarrow Z_{\mathbf{R}} \rightarrow \ell^{+} \ell^{-}$can be written as

$$
\begin{aligned}
\sigma\left(p p \rightarrow Z_{\mathbf{R}} \rightarrow \ell^{+} \ell^{-}\right)= & \frac{3}{s} \frac{\Gamma_{\text {tot }}}{M_{Z_{\mathbf{R}}}} \sum_{q} C_{q \bar{q}} \mathrm{BR}\left(Z_{R} \rightarrow q \bar{q}\right) \\
& \times \mathrm{BR}\left(Z_{\mathbf{R}} \rightarrow \ell^{+} \ell^{-}\right)
\end{aligned}
$$

where $\sqrt{s}$ is the centre-of-mass energy, $\Gamma_{\text {tot }}$ is the total width of $Z_{\mathbf{R}}, \operatorname{BR}\left(Z_{R} \rightarrow q \bar{q}\right)$ and $\mathrm{BR}\left(Z_{\mathbf{R}} \rightarrow \ell^{+} \ell^{-}\right)$are branching ratios of $Z_{\mathbf{R}}$ decaying to $q \bar{q}$ and $\ell^{+} \ell^{-}$respectively, $C_{q \bar{q}}$ are dimensionless partonic integrals with [62]

$C_{q \bar{q}}=\frac{4 \pi^{2}}{9} \int_{M^{2} / s}^{1} \frac{d x}{x}\left\{q(x) \bar{q}\left(\frac{M^{2}}{s x}\right)+\bar{q}(x) q\left(\frac{M^{2}}{s x}\right)\right\}$.

Their numerical values are calculated with the NNPDF [63] at $M=m_{Z_{\mathbf{R}}}$. We show in Fig. 6 the $\sigma\left(p p \rightarrow Z_{\mathbf{R}} \rightarrow \ell^{+} \ell^{-}\right)$ as the function of $m_{Z_{\mathbf{R}}}$ where the solid, dashed and dotted lines correspond to $g_{\mathbf{R}}=0.1,0.2$ and 0.3 respectively. The green and yellow bands are upper limits on the $\sigma(p p \rightarrow$ $\left.Z_{\mathbf{R}} \rightarrow \ell^{+} \ell^{-}\right)$at the $1 \sigma$ and $2 \sigma$ separately, given by the ATLAS collaboration [64] with $36.1 \mathrm{fb}^{-1}$ of proton-proton collision data collected at $\sqrt{s}=13 \mathrm{TeV}$. Since the $v_{\Phi}$ is constrained as $v_{\Phi}>18 \mathrm{TeV}$, there is a lower bound on the $Z_{\mathbf{R}}$ mass from the $Z-Z_{\mathbf{R}}$ mixing. By matching these two constraints, we find that the constraint from the dilepton search at the CERN LHC is stronger than that from the $Z-Z_{\mathbf{R}}$ mixing derived from diboson search at the LHC as well as the precision measurement of $Z$ boson mass only for $g_{\mathbf{R}}<0.121$ $\left(M_{Z_{R}}<4.37 \mathrm{TeV}\right)$.

\section{Conclusion}

Although the $U(1)_{\mathbf{R}}$ extension to the SM shares the same merit as the $U(1)_{\mathbf{B}-\mathbf{L}}$ extension of the SM on anomalies cancellations, its phenomenology was not investigated in detail in any reference except its effect in the vacuum stability of the SM Higgs. In this paper we constrained the parameter space of the model using the updated results of the $Z-Z^{\prime}$ mixing as well as the search of new resonance in dilepton channel at the LHC. Our investigation shows that the constraint from the dilepton search at the LHC is stronger than that from the $Z-Z_{\mathbf{R}}$ mixing derived from diboson search at the LHC and the precision measurement of $Z$ boson mass only for $g_{\mathbf{R}}<0.121$. We further studied the phenomenology of the $Z_{\mathbf{R}}$-portal dark matter and the possibility of generating active neutrino masses in the same model. It shows that the right-handed neutrino dark matter is self-consistent for its mass at near the resonant regime of $\hat{h}, \hat{s}$ and $Z_{\mathbf{R}}$ or for its mass being very heavy; the Majorana masses of active neutrinos can be generated from the modified type-II seesaw mechanism. It should be mentioned that the collider signature of $\hat{s}$ is Di-Higgs in various channels. We refer the reader to Ref. [65] for the Di-Higgs searches at various colliders for detail. $\hat{s}$ may also be searched at the LHC via the $p p \rightarrow \hat{s} \rightarrow Z_{\mathbf{R}} Z_{\mathbf{R}} \rightarrow \ell_{\alpha}^{+} \ell_{\alpha}^{-} \ell_{\beta}^{+} \ell_{\beta}^{-}$process if $m_{\hat{s}}>2 m_{Z_{\mathbf{R}}}$.

[Note added]: When this paper was being finalized, the paper [66] appeared, which partially overlaps with this one in discussing constraint from the precision measurement of $\mathrm{Z}$ boson mass, They use approximated formulae when do this analysis, while we present both full analytical and numerical results in this paper. Their study is largely complementary to ours.

Acknowledgements We thank to Huai-ke Guo for his help on numerical calculations and to Xiaohui Liu for helpful conversation. This work was supported by the National Natural Science Foundation of China under Grant No. 11775025 and the Fundamental Research Funds for the Central Universities.

Open Access This article is distributed under the terms of the Creative Commons Attribution 4.0 International License (http://creativecomm ons.org/licenses/by/4.0/), which permits unrestricted use, distribution, and reproduction in any medium, provided you give appropriate credit to the original author(s) and the source, provide a link to the Creative Commons license, and indicate if changes were made.

Funded by SCOAP ${ }^{3}$.

\section{References}

1. X.G. He, G.C. Joshi, H. Lew, R.R. Volkas, Phys. Rev. D 44, 2118 (1991). https://doi.org/10.1103/PhysRevD.44.2118 
2. S.F. King, S. Moretti, R. Nevzorov, Phys. Rev. D 73, 035009 (2006). https://doi.org/10.1103/PhysRevD.73.035009. arXiv:hep-ph/0510419

3. R.N. Mohapatra, R.E. Marshak, Phys. Rev. Lett. 44, 1316 (1980). https://doi.org/10.1103/PhysRevLett.44.1316. (Erratum: [Phys. Rev. Lett. 44, 1643 (1980)])

4. R.E. Marshak, R.N. Mohapatra, Phys. Lett. 91B, 222 (1980). https://doi.org/10.1016/0370-2693(80)90436-0

5. C. Wetterich, Nucl. Phys. B 187, 343 (1981). https://doi.org/10. 1016/0550-3213(81)90279-0

6. P. Fileviez Perez and M. B. Wise, Phys. Rev. D 82, 011901 (2010) Erratum: [Phys. Rev. D 82, 079901 (2010)] https://doi.org/10.1103/PhysRevD.82.079901, https://doi.org/10. 1103/PhysRevD.82.011901. arXiv:1002.1754 [hep-ph]

7. T.R. Dulaney, P. Fileviez Perez, M.B. Wise, Phys. Rev. D 83, 023520 (2011). https://doi.org/10.1103/PhysRevD.83.023520. arXiv:1005.0617 [hep-ph]

8. W. Chao, Phys. Lett. B 695, 157 (2011). https://doi.org/10.1016/j. physletb.2010.10056. arXiv:1005.1024 [hep-ph]

9. W. Chao, Phys. Rev. D 93(11), 115013 (2016) https://doi.org/10. 1103/PhysRevD.93.115013 arXiv:1512.06297 [hep-ph]

10. W. Chao, H. k. Guo and Y. Zhang, JHEP 1704, 034 (2017) https:// doi.org/10.1007/JHEP04(2017)034 arXiv:1604.01771 [hep-ph]

11. T. Appelquist, B.A. Dobrescu, A.R. Hopper, Phys. Rev. D 68, 035012 (2003). https://doi.org/10.1103/PhysRevD.68.035012. arXiv: hep-ph/0212073

12. A. Ekstedt, R. Enberg, G. Ingelman, J. Lgren, T. Mandal, JHEP 1611, 071 (2016). https://doi.org/10.1007/JHEP11(2016)071. arXiv:1605.04855 [hep-ph]

13. P. Langacker, Rev. Mod. Phys. 81, 1199 (2009). https://doi.org/10. 1103/RevModPhys.81.1199. arXiv:0801.1345 [hep-ph]

14. R.N. Mohapatra, Unification and supersymmetry: the frontiers of quark-lepton physics (Springer, Berlin, 2003)

15. J.L. Hewett, T.G. Rizzo, Phys. Rept. 183, 193 (1989). https://doi. org/10.1016/0370-1573(89)90071-9

16. P. Langacker, Phys. Rept. 72, 185 (1981). https://doi.org/10.1016/ 0370-1573(81)90059-4

17. W. Chao, M. Gonderinger, M.J. Ramsey-Musolf, Phys. Rev. D 86, 113017 (2012). https://doi.org/10.1103/PhysRevD.86.113017. arXiv:1210.0491 [hep-ph]

18. P. Minkowski, Phys. Lett. B 67, 421 (1977)

19. T. Yanagida, Workshop on unified theories. KEK Rep. 79-18, 95 (1979)

20. M. Gell-Mann, P. Ramond, R. Slansky, in Supergravity (North Holland, Amsterdam, 1979) eds. P. van Nieuwenhuizen, D. Freedman, p.315;

21. S. L. Glashow, in 1979 Cargese Summer Institute on Quarks and Leptons (Plenum Press, New York, 1980) eds. M. Levy, J.-L. Basdevant, D. Speiser, J. Weyers, R. Gastmans and M. Jacobs, p. 687

22. R. Barbieri, D.V. Nanopoulos, G. Morchio, F. Strocchi, Phys. Lett. B 90, 91 (1980)

23. R.N. Mohapatra, G. Senjanovic, Phys. Rev. Lett. 44, 912 (1980)

24. G. Lazarides, Q. Shafi, C. Wetterich, Nucl. Phys. B 181, 287 (1981)

25. W. Konetschny, W. Kummer, Phys. Lett. B 70, 433 (1977)

26. T.P. Cheng, L.F. Li, Phys. Rev. D 22, 2860 (1980)

27. G. Lazarides, Q. Shafi, C. Wetterich, Nucl. Phys. B 181, 287 (1981)

28. J. Schechter, J.W.F. Valle, Phys. Rev. D 22, 2227 (1980)

29. R.N. Mohapatra, G. Senjanovic, Phys. Rev. D 23, 165 (1981)

30. R. Foot, H. Lew, X. G. He and G. C. Joshi, Z. Phys. C 44, 441 (1989)

31. E. Witten, Phys. Lett. B 177, 324 (1982)

32. S.L. Adler, Phys. Rev. 177, 2426 (1969)

33. J.S. Bell, R. Jackiw, Nuovo Cimento A 60, 47 (1969)

34. W.A. Barden, Phys. Rev. 184, 1848 (1969)

35. R. Delbourgo, A. Salam, Phys. Lett. B 40, 381 (1972)

36. T. Eguchi, P.G.O. Freund, Phys. Rev. Lett 37, 1251 (1976)
37. L. Alvarez-Gaume, E. Witten, Nucl. Phys. B 234, 269 (1984)

38. P.P. Giardino, K. Kannike, I. Masina, M. Raidal, A. Strumia, JHEP 1405, 046 (2014). https://doi.org/10.1007/JHEP05(2014)046. arXiv: 1303.3570 [hep-ph]

39. W. Chao, arXiv:1601.06714 [hep-ph]

40. W. Chao, M. J. Ramsey-Musolf and J. H. Yu, Phys. Rev. D 93(9), 095025 (2016) https://doi.org/10.1103/PhysRevD.93. 095025 arXiv:1602.05192 [hep-ph]

41. P. Osland, A. A. Pankov and A. V. Tsytrinov, arXiv:1707.02717 [hep-ph]

42. C. Patrignani et al., Particle Data Group. Chin. Phys. C 40, 100001 (2016). (2017 update)

43. W. Chao, H. Zhang, Phys. Rev. D 75, 033003 (2007). https://doi. org/10.1103/PhysRevD.75.033003. arXiv: hep-ph/0611323

44. G. 't Hooft, Lecture given at Cargese Summer Inst., Cargese, France, 1979

45. P. Fileviez Perez, T. Han, G.Y. Huang, K. Wang, Phys. Rev. D 78, 015018 (2008). https://doi.org/10.1103/PhysRevD.78.015018. arXiv:0805.3536 [hep-ph]

46. W. Chao, Z.G. Si, Z.Z. Xing, S. Zhou, Phys. Lett. B 666, 451 (2008). https://doi.org/10.1016/j.physletb.2008.08.003. arXiv:0804.1265 [hep-ph]

47. W. Chao, Z.G. Si, Y.J. Zheng, S. Zhou, Phys. Lett. B 683, 26 (2010) https://doi.org/10.1016/j.physletb.2009.11.059. arXiv:0907.0935 [hep-ph]

48. N. Aghanim et al., Planck Collaboration. Astron. Astrophys. 594, A11 (2016). https://doi.org/10.1051/0004-6361/201526926. arXiv:1507.02704 [astro-ph.CO]

49. G. Arcadi, M. Dutra, P. Ghosh, M. Lindner, Y. Mambrini, M. Pierre, S. Profumo and F. S. Queiroz, arXiv:1703.07364 [hep-ph]

50. N. Okada, O. Seto, Phys. Rev. D 82, 023507 (2010). https://doi. org/10.1103/PhysRevD.82.023507. arXiv:1002.2525 [hep-ph]

51. W. Chao, Int. J. Mod. Phys. A 30(01), 1550007 (2015) https://doi. org/10.1142/S0217751X15500074. arXiv:1202.6394 [hep-ph]

52. W. Chao and M. J. Ramsey-Musolf, Phys. Rev. D 89(3), 033007 (2014) https://doi.org/10.1103/PhysRevD.89.033007. arXiv: 1212.5709 [hep-ph]

53. N. Okada and S. Okada, Phys. Rev. D 93(7), 075003 (2016) https://doi.org/10.1103/PhysRevD.93.075003. arXiv:1601.07526 [hep-ph]

54. N. Okada and S. Okada, Phys. Rev. D 95(3), 035025 (2017) https://doi.org/10.1103/PhysRevD.95.035025. arXiv:1611.02672 [hep-ph]

55. G. Bertone, D. Hooper, J. Silk, Phys. Rept. 405, 279 (2005). https:// doi.org/10.1016/j.physrep.2004.08.031. arXiv: hep-ph/0404175

56. J.R. Ellis, A. Ferstl, K.A. Olive, Phys. Lett. B 481, 304 (2000). https://doi.org/10.1016/S0370-2693(00)00459-7. arXiv:hep-ph/0001005

57. D. S. Akerib et al. [LUX Collaboration], Phys. Rev. Lett. 118(2), 021303 (2017) https://doi.org/10.1103/PhysRevLett.118.021303 arXiv:1608.07648 [astro-ph.CO]

58. A. Tan et al. [PandaX-II Collaboration], Phys. Rev. Lett. 117(12), 121303 (2016) https://doi.org/10.1103/PhysRevLett.117.121303. arXiv:1607.07400 [hep-ex]

59. G. K. Mallot, Int. J. Mod. Phys. A 15S1, 521 (2000) [eConf C 990809, 521 (2000)] https://doi.org/10.1142/ S0217751X00005309. arXiv:hep-ex/9912040

60. P. Agrawal, Z. Chacko, C. Kilic and R. K. Mishra, arXiv:1003.1912 [hep-ph]

61. C. Fu et al. [PandaX-II Collaboration], Phys. Rev. Lett. 118(7), 071301 (2017) https://doi.org/10.1103/PhysRevLett.118.071301. arXiv: 1611.06553 [hep-ex]

62. V. D. Barger and R. J. N. Phillips, REDWOOD CITY, USA: ADDISON-WESLEY (1987) 592 P. (FRONTIERS IN PHYSICS, 71) 
63. R.D. Ball et al., Nucl. Phys. B 867, 244 (2013). https://doi.org/10. 1016/j.nuclphysb.2012.10.003. arXiv:1207.1303 [hep-ph]

64. M. Aaboud et al. [ATLAS Collaboration], arXiv:1707.02424 [hepex]
65. T. Huang, J. M. No, L. Perni M. Ramsey-Musolf, A. Safonov, M. Spannowsky and P. Winslow, arXiv:1701.04442 [hep-ph]

66. T. Nomura and H. Okada, arXiv:1707.00929 [hep-ph] 